\title{
Abnormal slow wave mapping (ASWAM) - A tool for the investigation of abnormal slow wave activity in the human brain
}

\author{
Christian Wienbruch* \\ Department of Psychology, University of Konstanz, P.O. Box D23, D-78457 Konstanz, Germany
}

Received 15 January 2007; received in revised form 16 February 2007; accepted 18 February 2007

\begin{abstract}
Slow waves in the delta and theta frequency range, normal signs of deactivated networks in sleep stages, are considered 'abnormal' when prominent in the waking state and when generated in circumscribed brain areas. Structural cortical lesions, e.g. related to stroke, tumors, or scars, generate focal electric and magnetic slow wave activity in the penumbra. Focal concentrations of slow wave activity exceeding those of healthy subjects have also been found in individuals suffering from psychiatric disorders without obvious structural brain damage. Hence, identification and mapping of abnormal slow wave activity might contribute to the investigation of cortical indications of psychopathology.

Here I propose a method for abnormal slow wave mapping (ASWAM), based on a 5 min resting magnetoencephalogramm (MEG) and equivalent current dipole fitting to sources in the $1-4 \mathrm{~Hz}$ frequency band (delta) in anatomically defined cortical regions. The method was tested in a sample of 116 healthy subjects (59 males), with the aim to provide a basis for later comparison with patient samples. As to be expected, delta dipole density was low in healthy subjects. However, its distribution differed between genders with fronto-central > posterior dipole density in male and posterior dominance in female participants, which was not significantly related to either age or headsize. Results suggest that this method allows the identification of ASWA, so that comparison against $Z$-scores from a larger normal control group might assist diagnostic purposes in patient groups. As specific distributions seem to reflect differences between genders, this should be considered also in the analysis of patient samples. (C) 2007 Elsevier B.V. All rights reserved.
\end{abstract}

Keywords: Magnetoencephalography; Slow waves; Source localization; Delta band; Imaging; MEG

\section{Introduction}

Slow wave rhythms are a normal correlate of sleep stages, whereas in the waking state they are found in a variety of developmental and degenerative disorders, in toxic and metabolic encephalopathy and in other neurological conditions. If the neural generators are focally concentrated, slow wave rhythms appear in the vicinity of a structural lesion, that is, focal electromagnetic slow waves are generated in the surround of circumscribed lesions or neuronal tissue otherwise affected by pathology such as cerebral infarct, contusion, local infection, tumor, epileptic foci and subdural hematoma (Baayen et al., 2003; de Jongh et al., 2001a,b, 2002, 2003; Gallen et al., 1992, 1993; Tanaka et al., 1998; Vieth et al., 1998, 1996, 2001; Walter, 1936). Furthermore, reduced levels of neurotransmitters (e.g. actylcholine) can lead to an increase of delta activity (Neufeld

\footnotetext{
* Tel.: +49 7531 884610; fax: +49 7531884601 .

E-mail address: Christian.Wienbruch@uni-konstanz.de.
}

et al., 1994; Osipova et al., 2003; Riekkinen et al., 1990, 1991), consequently slowing of EEG is well known in Alzheimer's disease and cholinergic atrophy is believed to play an important role. Consequently, cholinergic monoaminergic blockade can partially reverse EEG slowing and (Dringenberg, 2000). Varying with changes in metabolism and blood flow consequent upon the insult (Meinzer et al., 2004; Strik et al., 2002; Vieth et al., 2001), abnormal slow wave activity has been attributed to a 'dysfunctional state' of the neuronal tissue (Lewine and Orrison, 1995). We may assume that 'de-afferented' neural networks cut off from major input sources produce focally generated slow waves.

In addition to neurological disease abnormal slow waves are observed in psychopathological conditions (Canive et al., 1996; Fehr et al., 2001, 2003; Fernandez et al., 2003; Wienbruch et al., 2003). Using a single equivalent current dipole model with band pass-filtered data in the delta $(1.5-4 \mathrm{~Hz})$ and theta $(4-8 \mathrm{~Hz})$ ranges, we (Fehr et al., 2003; Fernandez et al., 2002; Wienbruch et al., 2003) determined the origin of focal slow waves and a density function of focal generators. In psychiatric patients these density functions were circumscribed to regions generat- 
ing large-amplitude slow waves. Moreover, the localization of these foci distinguished diagnostic groups, with schizophrenia patients exhibiting more slow waves in association cortex, patients with Alzheimer's dementia being characterized by parietal foci (Fernandez et al., 2002), whereas a suppression of frontal activity compared to any of the comparison groups characterized depressive patients (Wienbruch et al., 2003). Given that the group-specific pattern of slow wave activity was not strongly affected by mental activation (Fehr et al., 2003), focal slow wave activity was assumed to indicate dysfunctions related to neuronal network communication caused by the psychological disorder. Thus, the mapping of abnormal brain oscillations might help to understand critical brain mechanisms in these disorders, and thereby support diagnosis as well as psychological and biological treatment development.

However, the benefit of abnormal slow wave mapping (ASWAM) in the study of 'dysfunctional' cortical network communication in clinical studies relies on testing of the method in healthy subjects. Here, we propose an improved method for ASWAM, extended from an initial method (Wienbruch et al., 2003) to allow Z-score based analysis of single subjects and group results of dipole density on both a voxel-scale and a scale of defined brain regions. The slow wave pattern determined from spontaneous magnetoencephalography (MEG) under resting conditions in a larger sample of healthy subjects should document the range of normal slow wave distribution and the potential variation with age and gender as a basis for the comparison with clinical groups. With this aim the present paper concentrates on the description of the method of ASWAM and its assessment in a sample of 116 healthy subjects, while further evaluation of the power of this method to distinguish between groups will be presented elsewhere.

MEG seems advantageous for the identification of focal slow wave activity as it allows the separation of focally generated slow waves from those with widespread sources, which is not the case with EEG. The same brain processes are differentially imaged by MEG and EEG and combined measurements of both MEG and EEG quantitatively illustrate this point (Eulitz et al., 1997). From a theoretical point of view, this may result from the nature of each signal. That is to say, EEG is strongly affected by the properties acting on the extracellular volume currents (e.g. conductivity) triggered mainly by postsynaptic potentials whereas MEG is thought to arise from the intracellular branch of this process, i.e., from the currents that flow from the dendrite to the soma. In a spherical volume conductor model MEG is only sensitive to currents flowing tangentially to the surface of the sphere. This is still (mainly) valid for realistic volume conductor models even if radial sources' contributions to the magnetic fields outside the volume conductor amounts to a degree of about $10 \%$. Widespread sources that extend across both sides of a sulcus have a strong radial contribution. However, as the activity in opposing walls of the sulci partially cancel each other, a weak tangential dipole source remains. Consequently, for focal sources located in sulci (which comprise two-third of the cortex) the signal-tonoise ratio is considerably higher for MEG measurements than for EEG measurements. Another advantage of MEG comes from the spatial attenuation of magnetic fields, which results in the situation that a subset of magnetic sensors can be used to scan a limited brain region for dipolar patterns. In contrast, with EEG the spread of volume currents affects even distant electrodes and thus, a focal activation cannot be modeled well if other activity is present, even if this activity is spatially dispersed.

\section{Method}

\subsection{Subjects}

Data from 116 individuals served to test the method of ASWAM. Subjects had been recruited as control subjects in different studies, in which MEG was recorded as described below. Thus, data acquisition was identical for all subjects. Across studies subjects were included as 'control' only, if they had never been treated for a neurological or psychiatric disorder and were not under medication (other than vitamins or aspirin or contraceptives). All subjects were right-handed as verified by the Edinburgh Handedness Questionnaire (Oldfield, 1971). The sample consisted of 59 males (mean age $31.7 \pm 10.9$, range 19-66 years) and 57 females (mean age $26.1 \pm 8.5$, range 17-64 years). Subjects were informed in detail about the measurement procedure, and a written consent was obtained from every subject prior to the measurement.

\subsection{Data acquisition}

Using a 148-channel whole-head neuromagnetometer (MAGNES $^{\text {TM }} 2500$ WH, 4D Neuroimaging, San Diego, USA) the MEG was measured during a 5 min resting period. Recordings were obtained in a supine position. Subjects were asked to relax but stay awake and not engage in any specific mental activity. They were further asked to fixate a mark on the ceiling of the magnetically shielded room throughout the recording in order to avoid eye- and head-movement. A video camera installed inside the chamber allowed monitoring the subject's behavior and compliance at any time throughout the measurement. The subject's index points and head shape were digitized with a Polhemus 3 Space ${ }^{\circledR}$ Fasttrack prior to each measurement (the nasion point, an anatomical landmark, and the left and right ear canal points served as index points and were used to define a right-handed coordinate system, called headframe coordinate system; the $x$-axis points to the front, the $y$-axis to the left and the $z$-axis to the top of the head). The subject's headsize was measured using three parameters: ANTERIOR, which is the $x$ coordinate of nasion point; LEFTRIGHT, which is the difference of $y$-coordinate of the left and right ear canal points; SUPERIOR, which is the maximum $z$-coordinate of all digitized head shape points. The subject's head position relative to the pickup coils of the sensor was estimated before and after each measurement.

\subsection{Data analysis - abnormal slow wave activity mapping (ASWAM)}

Slow wave generators were identified in a semi-automated procedure with the main steps application of noise reduction, decimation, digital band pass filters, and magnetic source imag- 
ing. Following noise reduction, data were screened for artifacts (e.g. eye blinks, muscle activity) by visual inspection and time periods without artifacts are selected. Data were then decimated by a factor of 16 (anti-alias filters are applied automatically in the same processing step) and digitally filtered separately for the delta $(1.5-4.0 \mathrm{~Hz})$ and theta $(4.0-8.0 \mathrm{~Hz}$; since results turned out to be identical for delta and theta frequencies, further steps of analysis and results refer to delta dipoles only) frequency band using a zero phase shift digital band pass filter (Butterworth filter of order 4).

Single equivalent current dipoles (ECD) were fitted for each time point in the selected artifact-free segments. Criteria applied for the selection of specific dipole solutions were goodness of fit $(\mathrm{GoF}$, to ensure the statistical significance of the source model) and dipole moment ( $Q$, to ensure focal nature of the underlying source based on neurophysiological consideration). Dipoles were selected by GoF >0.90 (de Munck et al., 2001) and $Q(10-100 \mathrm{nA} \mathrm{m})$, which is equivalent to $0.1-1 \mathrm{~cm}^{2}$ of activated cortex, and fulfills the assumption of a point source as assumed in the source model of an equivalent current dipole in a homogeneous sphere.

The dipole density was estimated within a volume with the anterior commissural (AC)-posterior commissural (PC) based coordinates $-X$ : posterior-anterior, $Y$ : right-left, and $Z$ : inferior-superior. The field of view was- $X$ : -12.0 to 10.0 (P-A), $Y$ : -11.0 to 11.0 (R-L), Z: -11.0 to 11.0 (I-S). The volume was divided into voxels with a size of $2 \mathrm{~cm} \times 2 \mathrm{~cm} \times 2 \mathrm{~cm}$. Hence, each volume comprises 1331 voxel. Within each voxel the selected ECD solutions were counted.

As artifact-free time periods varied between subjects, the dipole density was normalized by the subject's individual number of artifact-free (Nartfree) time points (normalized dipole density). Next, the distribution of dipole densities was analysed following two different strategies:

(a) The normalized dipole density $d$ was calculated for each subject $j$ within each voxel $i$ of the source volume (labeled voxel based dipole density, VBDD); then the logarithm of the normalized dipole density served to obtain a normal distribution across subjects (labeled LVBDD). The logarithm was computed for each voxel and each region except for those without fitted ECD (empty voxel), for which LVBDD it was set to 0. (Accordingly, such "empty" voxel did not contribute to averages in the following steps of analysis.) For further analysis and visualization LVBDD was $Z$-transformed for the single subjects using mean and standard deviation of LVBDD of the entire group of control subjects (labeled ZLVBDD).

The dipole density for the $i$ th voxel and the $j$ th subject is

$$
\begin{aligned}
& d_{i, j}=\left\{\begin{array}{ll}
\frac{\text { Ndipoles }_{i, j}}{\text { Nartfree }_{j}}, & \forall \text { Ndipoles }>0 \text { Nartfree }>0 \\
0
\end{array},\right. \\
& z_{i, j}= \begin{cases}\frac{\ln d_{i, j}-\mu_{i}}{\sigma_{i}}, & \forall d_{i, j}>0 \\
0\end{cases}
\end{aligned}
$$

$$
\begin{aligned}
\mu_{i} & =\frac{1}{\text { Nsubjects }} \sum_{j} \ln d_{i, j}, \\
\sigma_{i} & =\frac{1}{\text { Nsubjects }-1} \sqrt{\sum_{j}\left(\ln d_{i, j}-\mu_{i}\right)^{2}}
\end{aligned}
$$

Results were stored in the ANALYZE ${ }^{\mathrm{TM}} 7.5$ file format (http://www.mayo.edu/bir/PDF/ANALYZE75.pdf) and allowed the use of open source analysis and imaging tools.

(b) The second strategy determines the dipole density within anatomically defined brain areas (Fig. 1; Table A.1). For this region based dipole density (RBDD) schemata were generated for temporal, frontal, central and parietal parts of the brain within each hemisphere following the classification of the anatomical atlas provided with MRICRO's AAL (http://www.sph.sc.edu/comd/rorden/mricro.html) (see Appendix 1 for the list of anatomical structures included in each of the eight regions). Within these eight regions dipole density was estimated as the average of VBDD. As for VBDD, statistical analysis of RBDD was based on the logarithm of $Z$-transformed, region based mean and standard deviation from the entire sample (labeled ZLRBDD). A value of $0.5 /$ Nartfree was assigned to regions without fitted dipoles (i.e., 'empty' regions) before calculating the logarithm. (In contrary to (a) where it was absolutely necessary that empty voxels were assigned a value of zero, it was more convenient from a programmer's point of view to assign a value of $0.5 /$ Nartfree. This value is unique because the smallest possible value for a "non-empty" region is $1 /$ Nartfree. Therefore the statistics were not biased and the program does not have to keep track of empty regions.)

\subsection{Statistical analysis-mixed effects model for regional Z}

For the dependant variable ZLRBDD (for the sake of brevity termed $Z$ from hereon), variance components were estimated using PROC MIXED $\left(\mathrm{SAS}^{\circledR}\right.$ 9.1). Model 1 tested the fixed effects HEMI (comparing left- and right-hemispheric regions), REGION (comparing frontal, central, temporal, and parieto-occipital regions), GENDER (comparing male and female subjects) and the interaction of these factors $(H E M I \times$ GENDER, HEMI $\times$ REGION, REGION $\times$ GENDER and HEMI $\times$ REGION $\times$ GENDER) . The factor subject (ID), nested in GENDER, was specified as RANDOM as well as all interactions ID $\times$ HEMI and ID $\times$ REGION. Given its small covariance estimate the random effect ID $\times$ HEMI $\times$ REGION was discarded from all models. The covariance structure "VC = variance components" was chosen.

As visual inspection of raw data suggested a linear relationship between AGE and $Z$ (Fig. 2), the variable AGE was included as covariate (continuous predictor) in model 1. Since gender groups differed in age (females being younger than males: $t=3.1, p<0.003$ ) and headsize (females having smaller heads than males: LEFTRIGHT, $t=7.1, p<0.00001$; ANTERIOR, 

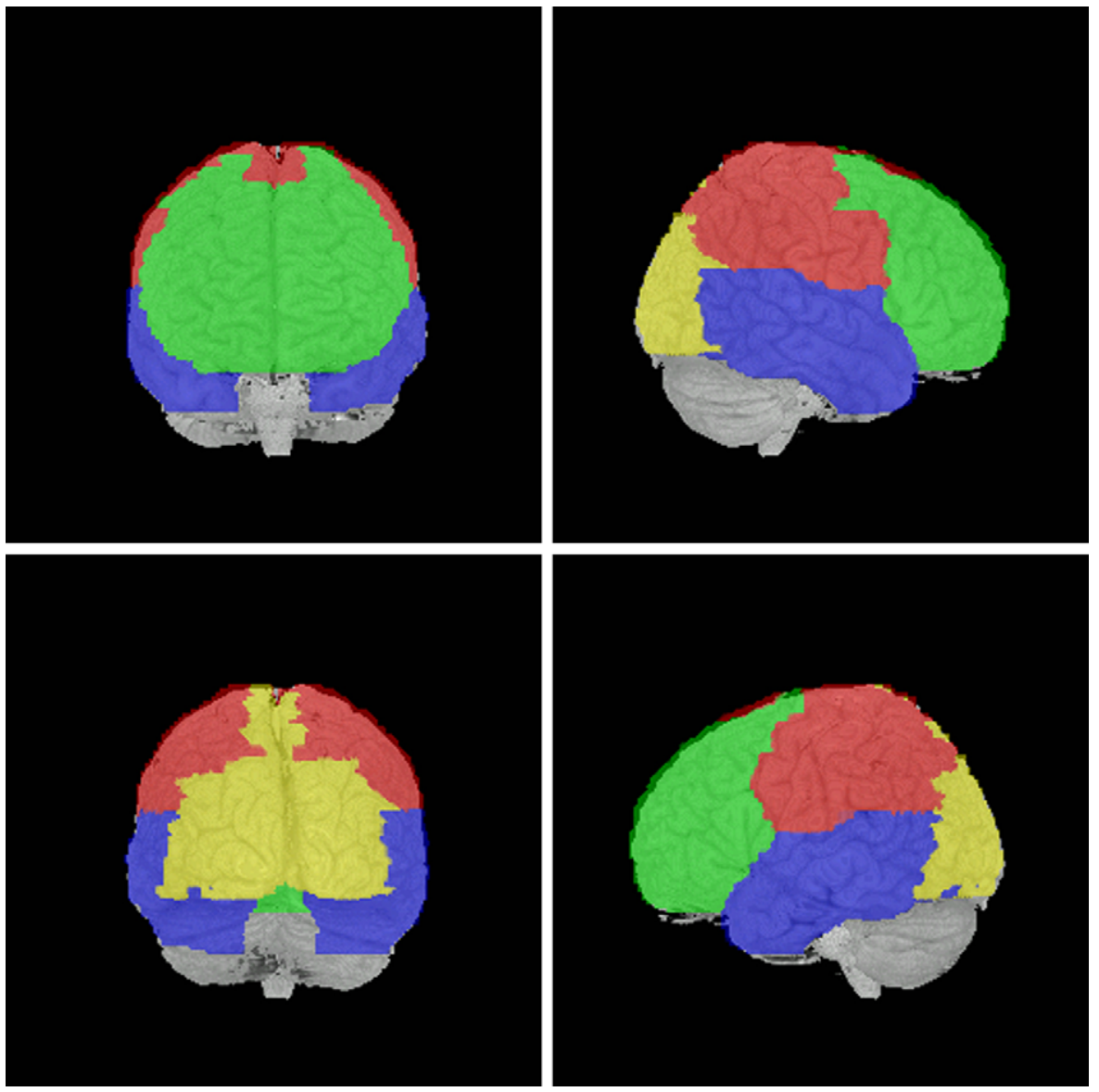

Fig. 1. Anatomically defined brain regions based on the AAL map (Tzourio-Mazoyer et al., 2002) included in mricro (http://www.sph.sc.edu/comd/rorden/ mricro.html): green $=$ frontal, blue $=$ temporal, yellow $=$ parieto-occipital, red $=$ central. (For interpretation of the references to color in this figure legend, the reader is referred to the web version of the article.)

$t=3.9, p<0.0002$; SUPERIOR, $t=3.3, p<0.002)$, separate analyses were conducted using age only (models $1 \mathrm{a}$ and $1 \mathrm{~b}$ ) and both, age and headsize parameters (model 2) as covariates of no interest. AGE and GENDER were hierachically separated and interactions of these two variables were not included. In order to account for the fact that covariates AGE, ANTERIOR, LEFTRIGHT, and SUPERIOR differed between groups, analy- ses were performed separately for females and males (Miller and Chapman, 2001). Linear regression models were calculated using PROC REG (SAS ${ }^{\circledR}$ 9.1) for significant interactions between continuous predictors and fixed effects. It should be noted that due to the large number of variables these correlations should not be over-interpreted, since correlations tend to become more easily significant with large $N$.
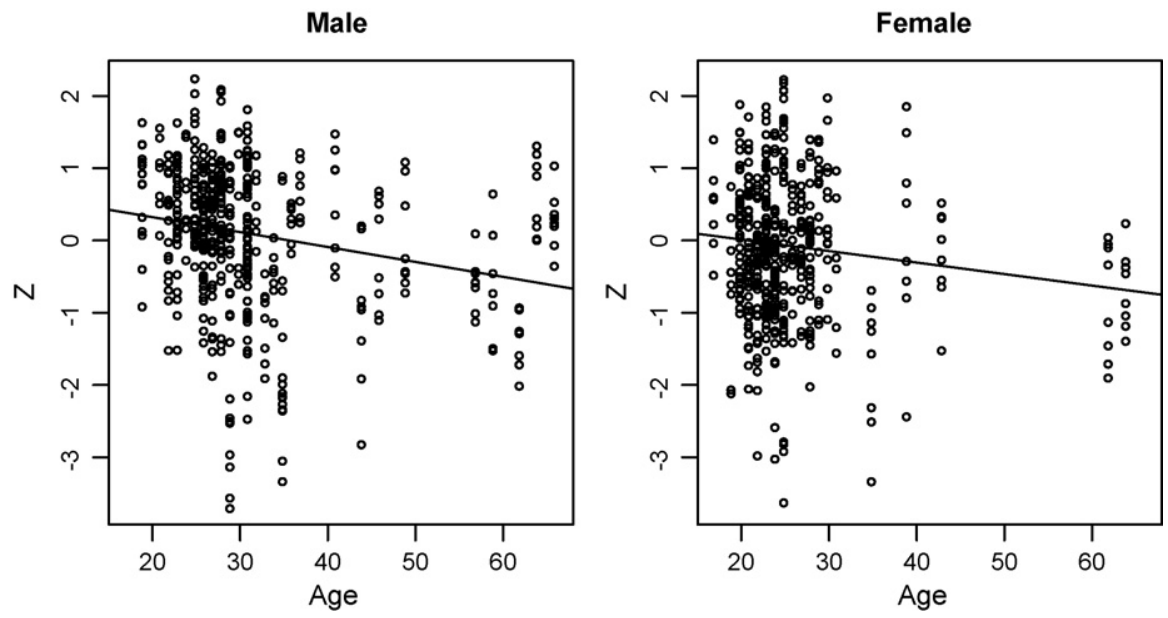

Fig. 2. Scatterplot of $Z$-scores (DELTA) vs. AGE for males (left column) and females (right column). 


\section{Results}

An average of $63,965 \pm 3178$ sample points per subject entered the analysis. The amount of artifact-free sample points was $49,339 \pm 10,314$ from which an average of $8937 \pm 2352$ had dipole fit solutions that met the selection criteria.

Within the entire sample, ASWA (despite slow wave activity in normal controls cannot be considered as abnormal the term ASWA is used as well) was small and evenly distributed. $Z$ did not vary between regions and only slightly between hemispheres (model 1: $F=3.7, p<0.06$ ). Mean $Z$ values of regions were always smaller than 0.4 (Fig. 3).

Still, the distribution of ASWA differed between males and females (see Fig. 4, GENDER: $F(1,113)=4.3, p<0.05$ ): male subjects displayed higher fronto-central ASWA than females $($ REGION $\times$ GENDER: $F(3,340)=12.3, p<0.0001)$. Post hoc tests confirmed the significant difference between males and females in frontal $(t=-3.95$, Tukey-Kramer adjusted $p<0.0001)$ and central $(t=-3.78$, Tukey-Kramer adjusted $p<0.0002)$ regions. In addition, the deviance from the mean distribution $(Z)$ decreased with increasing age (AGE: $F(1$, $338)=8.5, p<0.004)$. The linear correlation (PROG REG) confirmed an adjusted $r^{2}=0.016(F(1,455)=8.50, p<0.004)$ for female and an adjusted $r^{2}=0.048(F(1,471)=24.9, p<0.0001)$ for male participants.

Considering gender in separate analyses (PROC MIXED models $1 \mathrm{a}$ and $1 \mathrm{~b}$ ) confirmed this topographical pattern of ASWA. In females the main effect REGION $(F(3,156)=3.17$, $p<0.03$ ) confirmed the parieto-occipital $>$ frontal ASWA, without significant effect of AGE. In males the main effect $\operatorname{REGION}(F(3,165)=6.86, p<0.0002)$ resulted from the frontocentral dominance of ASWA, while a main effect AGE $(F(1$, $157)=4.37, p<0.04)$ verified lower ASWA in older participants. It seems noteworthy that least square estimates for ASWA differed only slightly between the models but much more importantly to a larger extend within the models (e.g. between regions).

Similarly, the gender-specific ASWA topography was substantiated after controlling for headsize by including parameters as continuous predictors (see above). Main effect and interactions with REGION remained significant for the female sample (REGION: $F(3,156)=3.17$, $p<0.03$; AGE $\times$ HEMI $\times$ REGION: $F(3,156)=2.69, p<0.05$; ANTERIOR $\times$ REGION: $F(3,156)=17.55, p<0.0001$; SUPERIOR $\times$ REGION $\quad F(3,156)=3.24, p<0.03$; ANTERIOR: $F(1,156)=3.80, p<0.06$; SUPERIOR: $F(1,156)=15.39$, $p<0.0002$ ), as well as for the male sample (REGION:
FL

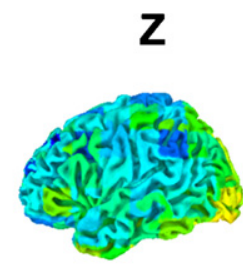

FR
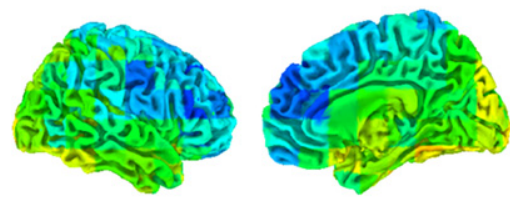

ML
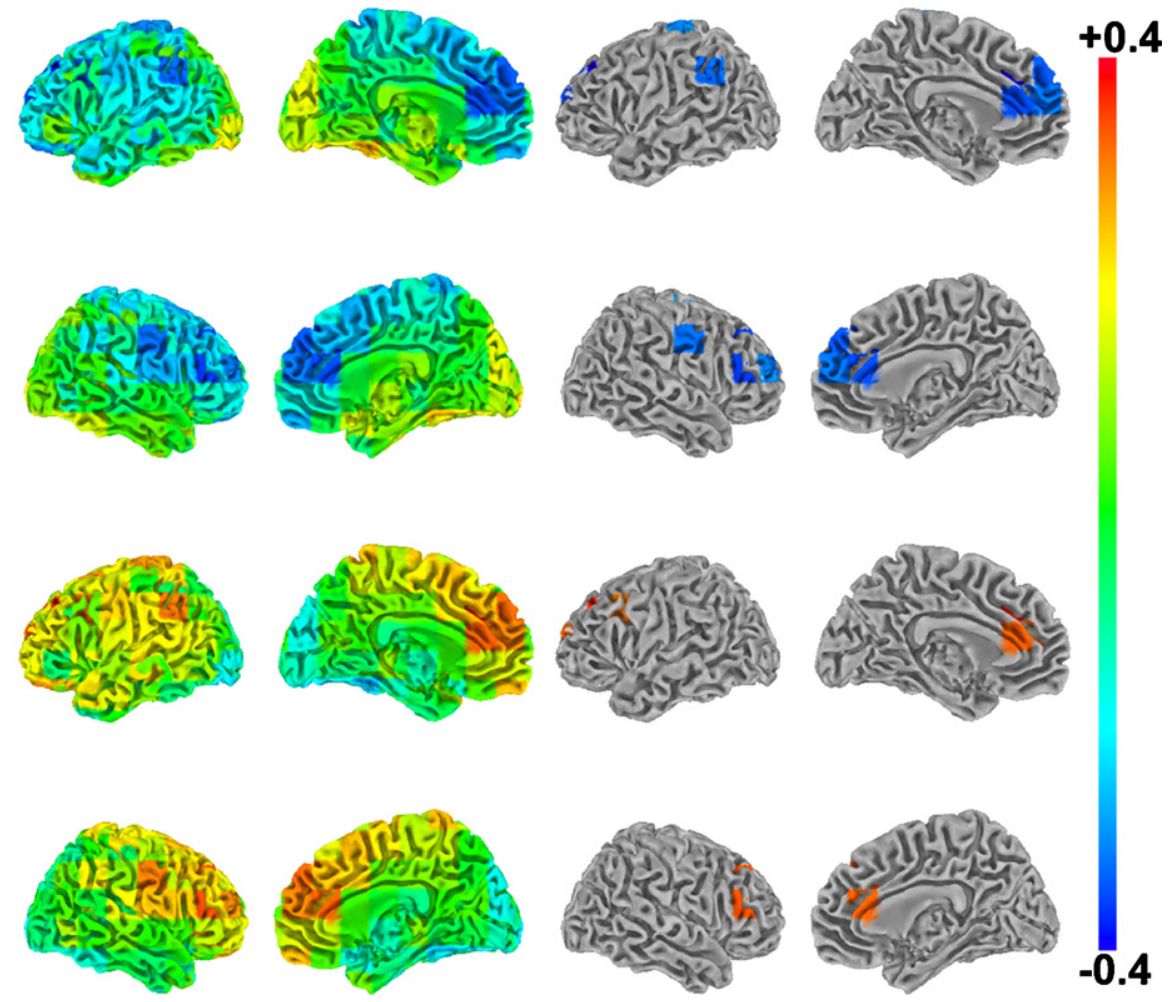

Fig. 3. Z-Scores for female (FL and FR) and male (ML and MR) subjects. FL and ML show the left FR and MR the right hemispheres (FL $=$ female left, FR $=$ female right, $\mathrm{ML}=$ male left, $\mathrm{MR}=$ male right). The outer surface of the hemispheres are displayed in the first and third column while the inner part is displayed in columns 2 and 4. Columns 1 and 2 show all $Z$-scores while columns 3 and 4 show only those regions where $t$-tests comparisons were significant $(p<0.05$; uncorrected for multiple comparisons). Colorscale ranges from -0.4 (dark blue) to 0.4 (red) while small (neutral) $Z$-scores have green colors. The segmented brain was calculated using the software package Caret (Van Essen et al., 2001), overlays of ASWA and the voxel based $t$-test results were done with AFNI/SUMA (Cox, 1996). (For interpretation of the references to color in this figure legend, the reader is referred to the web version of the article.) 
(a)
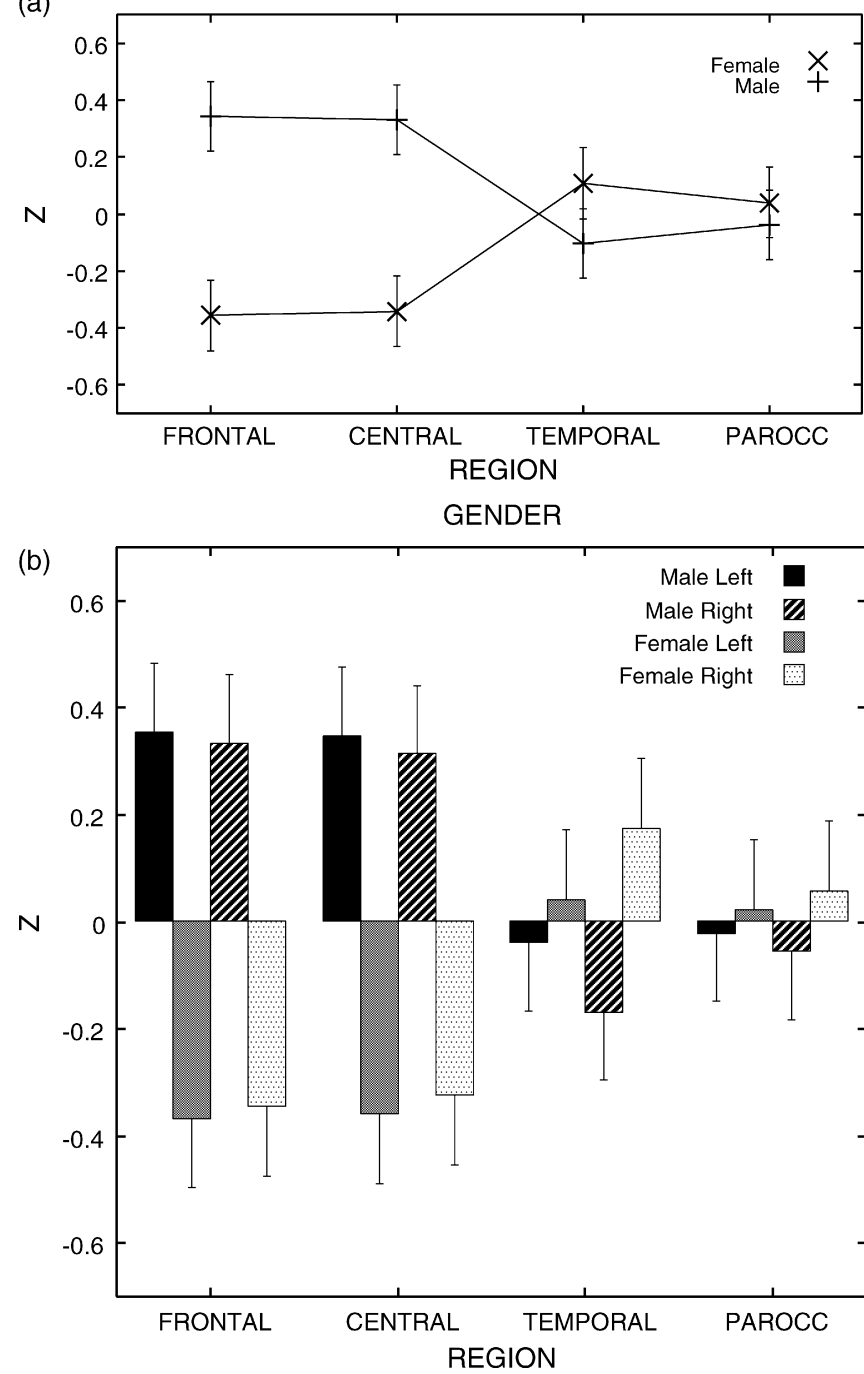

Fig. 4. Z-Scores in the DELTA band: (a) interaction REGION $\times$ GENDER and (b) interaction HEMI $\times$ REGION $\times$ GENDER. Males show larger $Z$-scores in fronto-central regions than females. Error bars indicate standard errors.

$F(3,165)=6.86, p<0.0002 ;$ ANTERIOR $\times$ REGION: $F(3$, $157)=21.06, \quad p<0.0002 ; \quad$ SUPERIOR $\times$ REGION: $\quad F(3$, $157)=7.03, \quad p<0.0003 ; \quad$ SUPERIOR $\times$ HEMI $\times$ REGION $:$ $F(3,157)=3.62, p<0.02$; AGE: $F(1,157)=4.37, p<0.04$; SUPERIOR: $F(1,157)=5.56, p<0.02)$. Linear single and multiple correlations between headsize parameters and dependent variables (see Tables A.2 and A.3 for specifications of the models, degrees of freedom, and results of the PROG REG models) were small (adjusted $r^{2}<0.1$ )—strengthening the assumption, that headsize did not significantly account for the gender differences in ASWA distribution.

\section{Discussion}

The aim of the present article is to introduce a method for mapping generators of electromagnetic slow wave activity on a voxel-scale and for anatomically defined cortical regions. The proposed method was applied to a sample of 116 healthy subjects in order to test the method and to disclose 'normal' variations of generator distribution with latent subject variables like gender or age. Results demonstrate the power of the method to disclose dipolar generators of slow wave activity, their distribution across cortical regions, and subtle differences in the slow wave distribution between subgroups of individuals. Further validation of the method must be complemented by its power to disclose abnormal slow wave clustering, that is, dipole densities exceeding those described for healthy subjects.

Though with low intensity, focal slow wave activity was present in the waking MEG in healthy individuals. Although slow wave activity in the waking state was addressed as indication of pathology in the introduction, its presence does not seem per se associated with abnormality. In healthy subjects, besides sleep, slow wave activity - though mainly in the 4-7 Hz theta band - can also be induced by a variety of mental activities including memory processes (Doppelmayr et al., 1998; Klimesch et al., 1996), arithmetic (Harmony et al., 1996), or processing demands (Asada et al., 1999). If aging correlates with an increase of small white-matter lesions and/or reduced neurotransmitter levels, one might expect an increase of slow wave activity with age. This slow wave activity can be expected to be of widespread nature, which means that it is only explained to a small extend by a focal source. In accordance the correlation between AGE and slow wave activity was found to be small and the direction of the relationship was opposite to the one expected. It can therefore be concluded that age does not increase ASWAM measures in healthy controls. Thus, we suggest that a distribution as found in the present sample of 116 subjects may represent a 'normal' range of neuronal network communication related to a 'normal' range of personality or psychological functioning, and that specific deviances from this normal distribution might be considered markers of abnormal neuronal network activity in psychiatric disorders.

Although the combination of data from control subjects (screened for absence of neurological and psychiatric disorders) served to describe the 'normal' slow wave distribution in healthy individuals, it may be questioned, whether the present sample of 116 participants represented the 'norm'. While the data acquisition procedure was identical for all participants and gender was equally distributed in this sample, subject recruitment did not meet standards of a representative sample, and age was not equally distributed among gender groups. Still, the results disclose substantial homogeneity, which encourages to consider the present sample as representing a 'normal' distribution of slow wave activity. However, this should and will be verified by continuing subject recruitment specifically designed to establish a representative sample of healthy subjects.

Although healthy male and female participants generated slow waves with similar intensity, the topography of slow wave generators differed with male subjects exhibiting more focal slow waves in fronto-central regions than females. As subsamples differed in age and as head circumference is often smaller in females than in males, the potential confounding effect of these variables was carefully analysed. Although an impact of both variables was indeed documented, it turned out to be small, and the gender-specific ASWA pattern was confirmed after controlling for age and headsize. In particular, it cannot be ruled 
out that a larger distance between the MEG sensors and the frontal cortex, as a consequence of smaller headsize in some female participants, reduce the sensitivity to detect anterior slow wave generators-hence, contributing to gender differences particularly in anterior ASWA. However, the small regressional coefficients and limited explained variance suggest that genderspecific ASWA cannot be interpreted as trivial consequence of different headsizes.

Gender differences in brain structure and function have been discussed at length (Cahill, 2006), but differences not related to gender-specific processing modes (like task or test performance) and not related to psychopathology are less frequently reported. With respect to spontaneous electromagnetic activity, higher percentage of left-frontal sleep spindles (Huupponen et al., 2002) or less pronounced long-range temporal correlations between occipito-parietal and frontal EEG-alpha generators were reported for females relative to males (Nikulin and Brismar, 2005) (in both reports, gender effects were independent of age)

Thus, if we consider gender differences in ASWA topography as an indication of genuine gender differences in neuronal network communication, analyses of abnormal slow wave activity should also consider this effect not only in psychiatric patients but also in normal controls, i.e., be performed separately for male and female groups. Another possibility to take the genderspecific ASWA topography into account is to estimate correction terms, e.g. subtraction of the least square estimates of $Z$ depending on gender, hemisphere and region as displayed in Fig. 4a and b. Gender-specific ASWA Z $Z$-scores of single subjects (e.g. patients) could then be considered to be abnormal if they are larger than 2 , meaning $Z$ is outside the range of 2 standard deviations of the $Z$-scores of the normal controls.

Finally, a straightforward control of the gender effect would define two control groups separate for gender. This would simplify the statistical models much as not only the factor GENDER disappears but also the continuous predictors for AGE, ANTERIOR, LEFTRIGHT, and SUPERIOR. Clinical testing of abnormal slow waves in single subjects is possible and results could be visualized using individual segmented brains. $Z$-scores for the normal controls, which are necessary to estimate $Z$-scores of individual subjects and source code to perform the analysis are available on request from the author.

In sum, testing a new method for the mapping of slow wave generators in a sample of $>100$ healthy subjects recommends the application of this method for the analysis of abnormal slow wave activity (ASWA) in subjects diagnosed with psychiatric disorders. The present results recommend a large group of normal control subjects from the age range 17-66 as basis to compare abnormal slow waves of patient samples with the aim to improve our understanding of abnormalities in neuronal network communication related to disorders, but also to assist diagnostic and prognostic purposes.

\section{Acknowledgements}

Research was supported by the German Research Foundation and the Volkswagen-Stiftung. I am grateful to U. Lommen and B. Awiszus, who accomplished all MEG recordings and data pre- processing, and to Brigitte Rockstroh and Thomas Elbert, who provided helpful suggestions and comments to earlier versions of the manuscript.

\section{Appendix A}

See Tables A.1-A.3.

Table A.1

Anatomical structures assigned to the four regions used for the calculation of RBDD

\begin{tabular}{|c|c|c|c|}
\hline \multicolumn{2}{|l|}{ Left } & \multicolumn{2}{|c|}{ Right } \\
\hline No. & AAL region & No. & AAL region \\
\hline \multicolumn{4}{|c|}{ Central } \\
\hline 1 & Precentral_L & 2 & Precentral_R \\
\hline 19 & Supp_Motor_Area_L & 20 & Supp_Motor_Area_R \\
\hline 33 & Cingulum_Mid_L & 34 & Cingulum_Mid_R \\
\hline 57 & Postcentral_L & 58 & Postcentral_R \\
\hline 59 & Parietal_Sup_L & 60 & Parietal_Sup_R \\
\hline 61 & Parietal_Inf_L & 62 & Parietal_Inf_R \\
\hline 63 & SupraMarginal_L & 64 & SupraMarginal_R \\
\hline 65 & Angular_L & 66 & Angular_R \\
\hline 69 & Paracentral_Lobule_L & 70 & Paracentral_Lobule_R \\
\hline \multicolumn{4}{|c|}{ Parieto-occipital } \\
\hline 35 & Cingulum_Post_L & 36 & Cingulum_Post_R \\
\hline 43 & Calcarine $\_\mathrm{L}$ & 44 & Calcarine_R \\
\hline 45 & Cuneus_L & 46 & Cuneus_R \\
\hline 47 & Lingual_L & 48 & Lingual_R \\
\hline 49 & Occipital_Sup_L & 50 & Occipital_Sup_R \\
\hline 51 & Occipital_Mid_L & 52 & Occipital_Mid_R \\
\hline 53 & Occipital_Inf_L & 54 & Occipital_Inf_R \\
\hline 67 & Precuneus_L & 68 & Precuneus_R \\
\hline \multicolumn{4}{|c|}{ Frontal } \\
\hline 3 & Frontal_Sup_L & 4 & Frontal_Sup_R \\
\hline 5 & Frontal_Sup_Orb_L & 6 & Frontal_Sup_Orb_R \\
\hline 7 & Frontal_Mid_L & 8 & Frontal_Mid_R \\
\hline 9 & Frontal_Mid_Orb_L & 10 & Frontal_Mid_Orb_R \\
\hline 11 & Frontal_Inf_Oper_L & 12 & Frontal_Inf_Oper_R \\
\hline 13 & Frontal_Inf_Tri_L & 14 & Frontal_Inf_Tri_R \\
\hline 15 & Frontal_Inf_Orb_L & 16 & Frontal_Inf_Orb_R \\
\hline 21 & Olfactory_L & 22 & Olfactory_R \\
\hline 23 & Frontal_Sup_Medial_L & 24 & Frontal_Sup_Medial_R \\
\hline 25 & Frontal_Mid_Orb_L & 26 & Frontal_Mid_Orb_R \\
\hline 27 & Rectus_L & 28 & Rectus_R \\
\hline 31 & Cingulum_Ant_L & 32 & Cingulum_Ant_R \\
\hline 71 & Caudate $\mathrm{L}$ & 72 & Caudate_R \\
\hline
\end{tabular}

Temporal

$\begin{array}{llll}17 & \text { Rolandic_Oper_L } & 18 & \text { Rolandic_Oper_R } \\ 29 & \text { Insula_L } & 30 & \text { Insula_R } \\ 37 & \text { Hippocampus_L } & 38 & \text { Hippocampus_R } \\ 39 & \text { ParaHippocampal_L } & 40 & \text { ParaHippocampal_R } \\ 41 & \text { Amygdala_L } & 42 & \text { Amygdala_R } \\ 55 & \text { Fusiform_L } & 56 & \text { Fusiform_R } \\ 73 & \text { Putamen_L } & 74 & \text { Putamen_R } \\ 79 & \text { Heschl_L } & 80 & \text { Heschl_R } \\ 81 & \text { Temporal_Sup_L } & 82 & \text { Temporal_Sup_R } \\ 83 & \text { Temporal_Pole_Sup_L } & 84 & \text { Temporal_Pole_Sup_R } \\ 85 & \text { Temporal_Mid_L } & 86 & \text { Temporal_Mid_R } \\ 87 & \text { Temporal_Pole_Mid_L } & 88 & \text { Temporal_Pole_Mid_R } \\ 89 & \text { Temporal_Inf_L } & 90 & \text { Temporal_Inf_R }\end{array}$

Definition of regions was based on the AAL map (Tzourio-Mazoyer et al., 2002) included in MRICRO (http://www.sph.sc.edu/comd/rorden/mricro.html). 
Table A.2

$r^{2}$ and adjusted $r^{2}$ for single and multiple correlation models

\begin{tabular}{|c|c|c|c|c|c|c|c|}
\hline Gender & Model & $r^{2}$ & Adjusted $r^{2}$ & DF model & DF error & $F$ & $p$ \\
\hline Female & $Z=\mathrm{AGE}$ & 0.0184 & 0.0162 & 1 & 454 & 8.50 & 0.0037 \\
\hline Female & $Z=$ ANTERIOR & 0.0139 & 0.0118 & 1 & 454 & 6.41 & 0.0117 \\
\hline Female & $Z=$ LEFTRIGHT & 0.0049 & 0.0027 & 1 & 454 & 2.24 & n.s. \\
\hline Male & $Z=\mathrm{AGE}$ & 0.0503 & 0.0483 & 1 & 470 & 24.90 & $<0.0001$ \\
\hline Male & $Z=$ ANTERIOR & 0.0001 & -0.0020 & 1 & 470 & 0.04 & n.s. \\
\hline Male & $Z=$ LEFTRIGHT & 0.0103 & 0.0082 & 1 & 470 & 4.90 & 0.0273 \\
\hline Male & $Z=$ AGE, ANTERIOR, LEFTRIGHT, SUPERIOR & 0.1023 & 0.0947 & 4 & 467 & 13.31 & $<0.0001$ \\
\hline
\end{tabular}

Table A.3

$r^{2}$ and adjusted $r^{2}$ for different multiple correlation models, within hemispheres and regions (model: $Z$ =AGE ANTERIOR LEFTRIGHT SUPERIOR)

\begin{tabular}{|c|c|c|c|c|c|c|c|c|}
\hline Gender & HEMI & REGION & $r^{2}$ & Adjusted $r^{2}$ & DF model & DF error & $F$-value & $p$ \\
\hline Female & Left & Frontal & 0.2395 & 0.1810 & 4 & 52 & 4.09 & 0.0058 \\
\hline Female & Left & Parocc & 0.4151 & 0.3701 & 4 & 52 & 9.23 & $<0.0001$ \\
\hline Female & Left & Temporal & 0.0994 & 0.0301 & 4 & 52 & 1.43 & n.s. \\
\hline Female & Right & Central & 0.3188 & 0.2664 & 4 & 52 & 6.08 & 0.0004 \\
\hline Female & Right & Frontal & 0.3571 & 0.3076 & 4 & 52 & 7.22 & 0.0001 \\
\hline Female & Right & Parocc & 0.3994 & 0.3532 & 4 & 52 & 8.65 & $<0.0001$ \\
\hline Male & Left & Frontal & 0.3171 & 0.2665 & 4 & 54 & 6.27 & 0.0003 \\
\hline Male & Left & Parocc & 0.1898 & 0.1298 & 4 & 54 & 3.16 & 0.0209 \\
\hline Male & Left & Temporal & 0.0241 & -0.0482 & 4 & 54 & 0.33 & n.s. \\
\hline Male & Right & Central & 0.3675 & 0.3206 & 4 & 54 & 7.84 & $<0.0001$ \\
\hline Male & Right & Frontal & 0.4481 & 0.4072 & 4 & 54 & 10.96 & $<0.0001$ \\
\hline Male & Right & Parocc & 0.2410 & 0.1847 & 4 & 54 & 4.29 & 0.0044 \\
\hline
\end{tabular}

\section{References}

Asada H, Fukuda Y, Tsunoda S, Yamaguchi M, Tonoike M. Frontal midline theta rhythms reflect alternative activation of prefrontal cortex and anterior cingulate cortex in humans. Neurosci Lett 1999;274:29-32.

Baayen JC, de Jongh A, Stam CJ, de Munck JC, Jonkman JJ, Trenite DG, et al. Localization of slow wave activity in patients with tumor-associated epilepsy. Brain Topogr 2003;16:85-93.

Cahill L. Why sex matters for neuroscience. Nat Rev Neurosci 2006;7:477-84.

Canive JM, Lewine JD, Edgar JC, Davis JT, Torres F, Roberts B, et al. Magnetoencephalographic assessment of spontaneous brain activity in schizophrenia. Psychopharmacol Bull 1996;32:741-50.

Cox RW. AFNI: software for analysis and visualization of functional magnetic resonance neuroimages. Comput Biomed Res 1996;29:162-73.

de Jongh A, Baayen JC, de Munck JC, Heethaar RM, Vandertop WP, Stam CJ. The influence of brain tumor treatment on pathological delta activity in MEG. Neuroimage 2003;20:2291-301.

de Jongh A, Baayen JC, de Munck JC, Puligheddu M, Stam CJ. Locations of sharp wave and slow wave generators in patients with brain tumors. In: Nowak H, Haueisen J, Gießler F, editors. BIOMAG 2002; 2002. p. 161-3.

de Jongh A, de Munck JC, Baayen JC, Jonkman EJ, Heethaar RM, van Dijk BW. The localization of spontaneous brain activity: first results in patients with cerebral tumors. Clin Neurophysiol 2001a;112:378-85.

de Jongh A, de Munck JC, Baayen JC, Jonkman EJ, Heethaar RM, van Dijk BW. Automatic magnetic source localization of spontaneous activity in patients with brain tumors. In: Nenonen J, Ilmoniemi RJ, Katila T, editors. BIOMAG 2000; 2001b. p. 431-4. de Munck JC, De Jongh A, Van Dijk BW. The localization of spontaneous brain activity: an efficient way to analyze large data sets. IEEE Trans Biomed Eng 2001;48:1221-8.

Doppelmayr M, Klimesch W, Schwaiger J, Auinger P, Winkler T. Theta synchronization in the human EEG and episodic retrieval. Neurosci Lett 1998;257:41-4.

Dringenberg HC. Alzheimer's disease: more than a 'cholinergic disorder'evidence that cholinergic-monoaminergic interactions contribute to EEG slowing and dementia. Behav Brain Res 2000;115:235-49.

Eulitz C, Eulitz H, Elbert T. Differential outcomes from magneto- and electroencephalography for the analysis of human cognition. Neurosci Lett 1997;227:185-8.

Fehr T, Kissler J, Moratti S, Wienbruch C, Rockstroh B, Elbert T. Source distribution of neuromagnetic slow waves and MEG-delta activity in schizophrenic patients. Biol Psychiatry 2001;50:108-16.

Fehr T, Kissler J, Wienbruch C, Moratti S, Elbert T, Watzl H, et al. Source distribution of neuromagnetic slow-wave activity in schizophrenic patients—effects of activation. Schizophr Res 2003;63:63-71.

Fernandez A, Arrazola J, Maestu F, Amo C, Gil-Gregorio P, Wienbruch $\mathrm{C}$, et al. Correlations of hippocampal atrophy and focal low-frequency magnetic activity in Alzheimer disease: volumetric MR imagingmagnetoencephalographic study. Am J Neuroradiol 2003;24:481-7.

Fernandez A, Maestu F, Amo C, Gil P, Fehr T, Wienbruch C, et al. Focal temporoparietal slow activity in Alzheimer's disease revealed by magnetoencephalography. Biol Psychiatry 2002;52:764-70.

Gallen CC, Schwartz BJ, Pantev C, Hampson S, Sobel D, Hirschkoff EC, et al., editors. Detection and localization of delta frequency activity in human strokes. Amsterdam: Excerpta Medica; 1992. 
Gallen CC, Sobel DF, Waltz T, Aung M, Copeland B, Schwartz BJ, et al. Noninvasive presurgical neuromagnetic mapping of somatosensory cortex. Neurosurgery 1993;33:260-8 [Discussion 8].

Harmony T, Fernandez T, Silva J, Bernal J, Diaz-Comas L, Reyes A, et al. EEG delta activity: an indicator of attention to internal processing during performance of mental tasks. Int J Psychophysiol 1996;24: 161-71.

Huupponen E, Himanen SL, Varri A, Hasan J, Lehtokangas M, Saarinen J. A study on gender and age differences in sleep spindles. Neuropsychobiology 2002;45:99-105.

Klimesch W, Doppelmayr M, Russegger H, Pachinger T. Theta band power in the human scalp EEG and the encoding of new information. Neuroreport 1996; 7:1235-40.

Lewine JD, Orrison Jr WW. Spike and slow wave localization by magnetoencephalography. Neuroimag Clin N Am 1995;5:575-96.

Meinzer M, Elbert T, Wienbruch C, Djundja D, Barthel G, Rockstroh B. Intensive language training enhances brain plasticity in chronic aphasia. BMC Biol 2004;2:20

Miller GA, Chapman JP. Misunderstanding analysis of covariance. J Abnorm Psychol 2001;110:40-8.

Neufeld MY, Rabey MJ, Parmet Y, Sifris P, Treves TA, Korczyn AD. Effects of a single intravenous dose of scopolamine on the quantitative EEG in Alzheimer's disease patients and age-matched controls. Electroencephalogr Clin Neurophysiol 1994;91:407-12.

Nikulin VV, Brismar T. Long-range temporal correlations in electroencephalographic oscillations: relation to topography, frequency band, age and gender. Neuroscience 2005;130:549-58.

Oldfield RC. The assessment and analysis of handedness: the Edinburgh inventory. Neuropsychologia 1971;9:97-113.

Osipova D, Ahveninen J, Kaakkola S, Jaaskelainen IP, Huttunen J, Pekkonen E. Effects of scopolamine on MEG spectral power and coherence in elderly subjects. Clin Neurophysiol 2003;114:1902-7.
Riekkinen P, Buzsaki G, Riekkinen Jr P, Soininen H, Partanen J. The cholinergic system and EEG slow waves. Electroencephalogr Clin Neurophysiol 1991;78:89-96

Riekkinen Jr P, Sirvio J, Riekkinen P. Relationship between the cortical choline acetyltransferase content and EEG delta-power. Neurosci Res 1990;8:12-20.

Strik C, Klose U, Kiefer C, Grodd W. Slow rhythmic oscillations in intracranial CSF and blood flow: registered by MRI. Acta Neurochir Suppl 2002;81:139-42.

Tanaka A, Kimura M, Yoshinaga S, Tomonaga M, Mizoguchi T. Quantitative electroencephalographic correlates of cerebral blood flow in patients with chronic subdural hematomas. Surg Neurol 1998;50:235-40.

Tzourio-Mazoyer N, Landeau B, Papathanassiou D, Crivello F, Etard O, Delcroix N, et al. Automated anatomical labeling of activations in SPM using a macroscopic anatomical parcellation of the MNI MRI single-subject brain. Neuroimage 2002;15:273-89.

Van Essen DC, Drury HA, Dickson J, Harwell J, Hanlon D, Anderson CH. An integrated software suite for surface-based analyses of cerebral cortex. J Am Med Inform Assoc 2001;8:443-59.

Vieth J, Kober H, Ganslandt O, Möller M, Kamada K. The clinical use of MEG activity associated with brain lesions. In: Nenonen J, Ilmoniemi RJ, Katila T, editors. BIOMAG 2000; 2001. p. 387-94.

Vieth JB, Kober H, Grummich P. Sources of spontaneous slow waves associated with brain lesions, localized by using the MEG. Brain Topogr 1996;8:215-21.

Vieth J, Kober H, Kamada K, Ganslandt O. Normal and abnormal MEG activity in border zones of brain lesions. In: Koga Y, editor. Brain topography today. Amsterdam: Elsevier; 1998. p. 39-46.

Walter G. The location of cerebral tumors by electroencephalography. Lancet 1936;8:305-8.

Wienbruch C, Moratti S, Elbert T, Vogel U, Fehr T, Kissler J, et al. Source distribution of neuromagnetic slow wave activity in schizophrenic and depressive patients. Clin Neurophysiol 2003;114:2052-60. 